\title{
INFLUENCE OF NONCONDENSABLE GAS TO CONDENSATION OF WATER IN A NANOSCALE SPACE USING MOLECULAR DYNAMICS SIMULATION
}

\author{
$\mathrm{Li} \mathrm{Li}^{*} \mathcal{E}$ Xiaoze Du
}

\begin{abstract}
MOE Key Laboratory of Condition Monitoring and Control for Power Plant Equipment, North China Electric Power University, Beijing 102206, China

*Address all correspondence to: Li Li, MOE Key Laboratory of Condition Monitoring and Control for Power Plant Equipment, North China Electric Power University, Beijing 102206, China; Tel./Fax: +86 106177 3958, E-mail: doubleli@ncepu.edu.cn
\end{abstract}

Original Manuscript Submitted: 5/10/2019; Final Draft Received: 7/8/2019

The existence of noncondensable gases has a great effect on the condensation heat transfer coefficient. It is necessary to exclude noncondensable gases and maintain the vacuum degree to improve heat transfer in some industrial phase-change heat exchangers. Nevertheless, to better control the temperature needed to increase thermal resistance to decrease or adjust the heat transfer, using noncondensable gases is an effective method. Understanding heat transfer and dynamics characteristics of noncondensable gases at nanoscale are of great interest in both theoretical and practical applications. In the present study, the influence of noncondensable gases to phase change in confined nanoscale space was investigated by using molecular dynamics simulation. Vapor nitrogen was used as the noncondensable gas put into the working fluid region that contains the liquid and vapor water. The temperature and distribution of the density of working fluid was obtained and the trajectories of some water molecules and nitrogen molecules were tracked. As time passes, more and more water molecules condense at the cold wall, whereas a certain number of nitrogen molecules fluctuate in the working fluid region beside the accumulated nitrogen molecules at the cold end to resist the heat transfer and increase the temperature difference. The results revealed the influence of noncondensable gases to phase change from the nanoscale aspect. The enhancement of heat transfer could be realized and controlled through the regulation of the noncondensable gases in the working fluid.

KEY WORDS: noncondensable gas, condensation, molecular dynamics simulation

\section{INTRODUCTION}

Phase change heat transfer plays an important role in a variety of high-efficiency heat exchangers because of the high heat transfer coefficient. A small amount of noncondensable gases mixed in pure vapor has a great effect on the condensation heat transfer coefficient. The noncondensable gases are considered a negative factor for improving heat transfer in some industrial phase-change heat exchangers such as air condensers, air conditioners, and standard heat pipes. It is necessary to try to exclude the noncondensable gases and keep the vacuum degree to guarantee the heat transfer efficiency. Nevertheless, for better control of temperature or for 


\begin{tabular}{|c|c|c|c|}
\hline \multicolumn{4}{|c|}{ NOMENCLATURE } \\
\hline$q$ & charge, $\mathrm{C}$ & Subsc & ipts \\
\hline$r$ & $\begin{array}{l}\text { distance between two } \\
\text { atoms, } \AA\end{array}$ & $\begin{array}{l}a, b \\
f\end{array}$ & $\begin{array}{l}\text { different atom } \\
\text { fluid }\end{array}$ \\
\hline$x, y, z(\mathrm{X}, \mathrm{Y}, \mathrm{Z})$ & $\begin{array}{l}\text { direction (coordinate } \\
\text { axis) }\end{array}$ & $\begin{array}{l}\mathrm{i}, \mathrm{j} \\
\mathrm{m}, \mathrm{n} \\
\mathrm{s}\end{array}$ & $\begin{array}{l}\text { different charge site } \\
\text { different oxygen atom } \\
\text { solid }\end{array}$ \\
\hline Greek Symbols & & $\mathrm{CuCu}$ & copper-copper \\
\hline$\sigma$ & $\begin{array}{l}\text { finite distance at which } \\
\text { the interatomic potential }\end{array}$ & & $\begin{array}{l}\text { hydrogen charge site in } \\
\text { TIP4P/2005 force field }\end{array}$ \\
\hline $\mathcal{\varepsilon}$ & $\begin{array}{l}\text { is zero, } \AA \\
\text { depth of the L-J } \\
\text { potential, J }\end{array}$ & $\begin{array}{l}\mathrm{M} \\
\mathrm{NN}\end{array}$ & $\begin{array}{l}\text { additional charge site in } \\
\text { TIP4P/2005 force field } \\
\text { nitrogen-nitrogen }\end{array}$ \\
\hline$\varphi$ & effective pair potential, $\mathrm{J}$ & $\mathrm{OO}$ & oxygen-oxygen \\
\hline
\end{tabular}

overtemperature protection, which needs to increase thermal resistance to decrease or adjust the heat transfer between heat source and heat sink, the presence of noncondensable gas is an effective method, such as in the gas-loaded or variable conduction heat pipe (Edwards and Marcus, 1972; Rohani and Tien, 1973; Edom and Vlassov, 2001). On the basis of a standard heat pipe, noncondensable gas is added into the working fluid. At different power, various fraction ratios of noncondensable gas are driven to adjust the volume of condenser by different temperatures and corresponding pressure to block various portions of the condenser. In addition, under high working temperature that far exceeds the tolerance limits of materials, the existence of cooling gas between the high temperature heat source and working part may increase the thermal resistance to decrease the thermal damage of high heat to working materials, such as the film cooling technology in the turbine blade. Understanding the dynamic characteristics of noncondensable gases similar to fouling control (Kim, 2019) is very important not only for improving the condensation heat transfer coefficient but also for the temperature controlling and design of heat insulation.

After the first experimental research on the effect of noncondensable gases to condensation, many experiments and simulations were performed in this region. Many macroscale experiments of condensation on vertical or horizontal surfaces under forced or natural convection were performed to study the effect of noncondensable gases such as air, nitrogen, argon, and so on. The results of these studies show that noncondensable gases would impede the heat transfer of condensation. There are detailed descriptions about the methods and results in review literature (Huang et al., 2015). In recent years, many experiments and simulations have continued working on the effect of noncondensable gases from a point of view disadvantageous to condensation (Ren et al., 2015; Yi et al., 2016; Zhou et al., 2017). Literatures (Sugimoto et al., 2011; Leriche et al., 2012; Lee et al., 2017) have experimentally and numerically studied the effect of noncondensable gases on heat transfer characteristics and the performance of variable conductance heat pipes to adjust the heat transfer depending on the heat load. 
The experiments and simulations in the literatures, mentioned earlier, related to the effect of the noncondensable gas basically focused on the macroscale rules of flow and heat transfer. With the development of science and technology, understanding the mechanism of nanoscale and microscale phase change is crucially important to control the heat transfer for better thermal management. Besides, with the improvement of manufacturing level, more and more micro- and nanoscale heat exchangers are produced. The deep understanding of phase change and the effect of noncondensable gases inside the micro- and nanoscale heat exchangers from a microscopic perspective can contribute to the further development of theory and technology.

The vaporization and condensation occurring at nanoscale has always been a hot topic in both theoretical and experimental fields in the last few decades. Li et al. (2017) and Zhang et al. (2017) applied Lattice Boltzmann simulation to investigate the condensation in the presence of a noncondensable gas from the microscale. The presence of noncondensable gas obviously reduces the condensation mass rate and the heat flux compared to the pure vapor. The molecular dynamics (MD) method has been widely implemented to research the interfacial thermal transport (Cahill et al., 2003). The statistical thermodynamic information and heat transfer characteristics are obtained by solving Newton's equation of motion of atoms or molecules in a system. Literatures (Nagayama et al., 2010; Morshed et al., 2011; Yu and Wang, 2012; Seyf and Zhang, 2013; Diaz and Guo, 2017a,b) carried out MD simulation to understand the evaporation behavior of explosive and normal boiling of argon on different walls. MD simulation was employed by Li et al. (2016) to study the condensation of argon on smooth and nanostructure copper surface. Both vaporization and condensation of argon on platinum surface were simulated by Liang et al. (2017) and Wang et al. (2007). Yang and Pan (2005) investigated the evaporation of a thin water layer into a vacuum using the MD method through FORTRAN code. The TIP4P model was used for intermolecular potential of water in the study. The molecular behavior near and in the interface region and the effect of a hydrogen bond was studied in detail. Diaz and Guo (2019) employed the MD simulation to study the improvement of the critical heat flux in water boiling heat transfer through adding graphene to substrates. Hu and Sun (2012) studied the effect of nanopatterns on Kapitza resistance at a water-gold interface during boiling. The TIP4P/Ew model was used to calculate the potential of water. Louden et al. (2013) researched the condensation coefficient of water using the SPC/E model for intermolecular potential of water. Mao and Zhang (2014) studied the rapid boiling of pure water on a copper surface; the TIP4P model is also used in this simulation. Arismendi-Arrieta et al. (2014) calculated the structural and transport properties of pure liquid water using MD simulation. They found that by adjusting the molecular dipole moment of the Niesar, Clementi and Corongiu (NCC) model to the value of the TIP4P/2005 water model, the values for self-diffusion and viscosity coefficients are in better agreement with experiment, compared to the values obtained with the original NCC model.

Most of the aforementioned literature using the MD simulation focused on studying the vaporization or condensation of the pure working fluids. As mentioned before, experiments and simulations about the phase-change phenomenon with impurity or noncondensable gas are focused on macroscale instead of microscale or nanoscale. Therefore, the present study concentrates on the effect of noncondensable gases to the nanoscale phase change and heat transfer using classical MD simulation, especially on the condensation on the cold wall. Water is chosen as the working fluid and copper is used as the material of the solid wall. Nitrogen is designed to add in the working fluid region as the noncondensable gas. Evaporation and condensation of the heated and cooled water molecule without or with different numbers of nitrogen molecules in the processing are studied. The research is considered to provide a better understanding of the

Volume 27, Issue 1, 2020 
molecular level phase change process with impurity or noncondensable gas. Regulation of the noncondensable gas leads to better control or enhancement of heat transfer.

\section{SIMULATION METHOD}

The molecular simulation system consists of solid copper walls, water molecules, and nitrogen molecules with lattice structure of face-centered cubic (FCC) unit cells. Solid copper walls are located at the bottom and top ends to work as heat source and heat sink. Water molecules including liquid and vapor phases were arranged in the working fluid region. Keeping the number of water molecules constant, cases without vapor nitrogen and with a number of vapor nitrogen molecules were designed in the system to study the influence of nitrogen as a noncondensable gas to phase-change water. The length (in the $x$ direction) equals the width (in the $y$ direction) of the simulation box with periodic boundary conditions applied in the two directions. The height (in the $z$ direction) of the box was the summation of the heights of all the copper walls and working fluid region.

As shown in Fig. 1, for both the hot and cold walls on the bottom and top of the simulation box, solid copper walls were divided into fixed wall, heat source/sink wall, and heat conduction wall. To prevent the thermal energy of the entire system from the losing and flying away of atoms, copper atoms in the fixed wall were fixed without vibration. Thermostats were used to maintain constant temperature of the heat source and sink walls that are adjacent to the fixed walls. A constant high-temperature boundary condition was placed on the hot wall to supply the heat to the liquid water for the vaporization. The conduction wall at the bottom side was implemented to conduct heat from the heat source to the liquid water. Liquid phase working fluid molecules absorb thermal energy from the heat source and improve their velocities to overcome the attractive force from the copper wall, then translate to vapor phase and transfer the heat to the

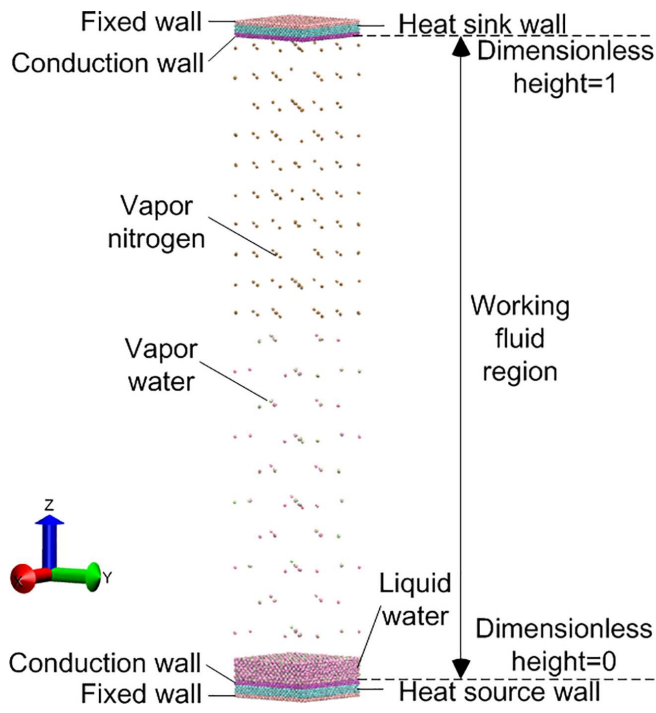

(a)

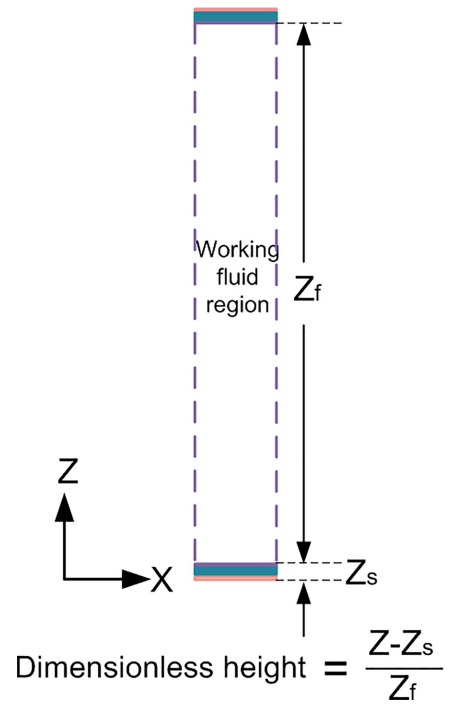

(b)

FIG. 1: Schematic diagram of simulation domain: (a) orthographic view, (b) the principle of the dimensionless height 
top side through continuous collisions among molecules. Molecules with high energy condense at the bottom surface of the conduction wall adjacent to the heat sink wall to transfer the energy to the cold side. The conduction wall at the top side was implemented to absorb thermal energy from the condensed water to the heat sink. The constant low temperature boundary condition was imposed on the cold wall to absorb the heat.

The liquid water region was in contact with the conduction wall of the hot wall side, which includes 13,043 liquid water molecules with the arrangement of FCC unit cells. Above the region of the liquid water, 160 vapor water molecules were placed. In Case 0, only water molecules in the working fluid region. In Case 1, 160 vapor nitrogen molecules were added above the vapor water in the working fluid region (the number of vapor nitrogen molecules is 350 and 500 in Case 2 and 3, respectively) to research the effect of noncondensable gases to the condensation of water. What needs to be explained in advance is that the value of the whole height of the working fluid region was divided to calculate the dimensionless height of fluid molecules in the $z$ direction for a direct comparison of the position of different fluid molecules. Considering the change of positions was limited in the working fluid region, the height of the working fluid region $\left(Z_{\mathrm{f}}\right)$ but not the height of the whole computational domain box was chosen as the denominator. The two positions of dimensionless height equals 0.0 and 1.0 located at the upper surface of hot conduction wall and the lower surface of cold conduction wall, respectively, which were labeled in Fig. 1(a). The computing method of the dimensionless height was given in Fig. 1(b).

The noncondensable gas of vapor nitrogen molecules was considered a dual-atom molecule without the internal bonding interaction. The interatomic interaction between copper-copper and nitrogen-nitrogen were described by the standard 12/6 L-J potential function (as shown in Eq. 1) with a cutoff distance of $12 \AA$, which had already shown to be a simple and reliable potential function. The energy $\varepsilon$ and length $\sigma$ parameters for same atomic interactions in L-J potential function was calculated through unit conversion; refer to the data $\left(\varepsilon_{\mathrm{CuCu}}=65.63 \times 10^{-21} \mathrm{~J}\right.$; $\sigma_{\mathrm{CuCu}}=0.233 \mathrm{~nm} ; \varepsilon_{\mathrm{NN}}=1.3932 \times 10^{-21} \mathrm{~J} ; \sigma_{\mathrm{NN}}=0.3623 \mathrm{~nm}$ ) in literature (Jia et al., 2012; Zou et al., 2010):

$$
\varphi(r)=4 \varepsilon\left[\left(\frac{\sigma}{r}\right)^{12}-\left(\frac{\sigma}{r}\right)^{6}\right]
$$

For the vapor and liquid water molecule, a four-site water model (TIP4P) with improved parameters called TIP4P/2005 (Abascal and Vega, 2005; Péreza and Rubiob, 2011) was adapted to model the intermolecular potential interaction between water molecules. The TIP4P/2005 is a rigid planar model with $\mathrm{OH}$ bond length equal to $0.9572 \AA$ and bond angle between two $\mathrm{OH}$ bonds equal to $104.52^{\circ}$. The intermolecular potential interaction consists of the LennardJones interaction between oxygen atoms and Coulomb force between hydrogen atoms and the additional site located $0.1546 \AA$ away from the oxygen atom along the bisector of the $\mathrm{HOH}$ angle. The calculation formula could be written as

$$
\varphi\left(r_{i, j}\right)=\sum_{m, n<m} 4 \varepsilon_{\mathrm{OO}}\left[\left(\frac{\sigma_{\mathrm{OO}}}{r_{m n}}\right)^{12}-\left(\frac{\sigma_{\mathrm{OO}}}{r_{m n}}\right)^{6}\right]+\frac{1}{4 \pi \varepsilon_{0}} \sum_{i, j<i} \frac{q_{i} q_{j}}{r_{i j}}
$$

in which $r_{m n}$ denotes the distance between oxygen atoms of two water molecules and $r_{i j}$ denotes the distance between charged sites of two water molecules. The long-range Coulombic force is computed by the particle-particle, particle-mesh technique. Some parameters in the TIP4P/2005 model used for same atomic interactions calculated through unit conversion refer to the data $\left(\varepsilon_{\mathrm{OO}}=0.7749 \mathrm{~kJ} / \mathrm{mol} ; \sigma_{\mathrm{OO}}=3.1589 \AA ; q_{H}=0.5564 e ; q_{M}=-2 q_{H}\right)$ in literature (Péreza

Volume 27, Issue 1, 2020 
and Rubiob, 2011). Lorentz-Berthelot combining rule was used to calculate related parameters between different atoms, namely, $\sigma_{a b}=(1 / 2)\left(\sigma_{a a}+\sigma_{a b}\right)$ and $\varepsilon_{a b}=\sqrt{\varepsilon_{a a} \varepsilon_{b b}}$.

The simulation was performed by using the large-scale atomic/molecular massively parallel simulator distributed by Sandia National Laboratories. And the VMD software was used to obtain the visual snapshots and position data of molecules. For the entire simulation, the time step was set as $0.1 \mathrm{fs}$. The simulation process can be divided into three consequent stages. The first stage was to perform the relaxation in which the conjugate gradient algorithm was performed and the number of steps was limited to 100,000 steps. Initial temperature of the liquid and vapor molecules was set as $273.16 \mathrm{~K}$. The canonical ensemble and the microcanonical ensemble were used to simulate 100,000 steps sequentially by keeping constant number, volume, temperature, or energy of working fluid in the simulation at the second stage. The heat source wall was set to a $700 \mathrm{~K}$ high temperature to supply heat and the heat sink wall was set to $273 \mathrm{~K}$ for the absorption of heat. The heat conduction walls on the two ends contacting the heat source/sink walls and working fluid were set as an adiabatic wall to impede heat exchange between the heat source/sink and working fluid. The working fluid molecules showed stable profiles of temperature evolutions, which indicated the thermal equilibrium. Then the adiabatic control of the conduction walls was removed at the third stage, which allowed the heat transfer from hot wall to cold wall through phase change and moving of the working fluid molecules. Cases without nitrogen and with nitrogen in addition to the water were considered throughout the simulations. The average temperatures and numbers and trajectories of liquid/vapor molecules were recorded to analyze the transport phenomenon.

\section{RESULTS AND DISCUSSION}

\subsection{Effect of Noncondensable Gas}

Figure 2 illustrates the average temperature of the water molecules including liquid and vapor phases in the cases of with nitrogen and without nitrogen. As mentioned before, the time coordination in this figure and the later figures is counted from the beginning of the working fluid being heated by the conduction wall connected with heat source after removing the adiabatic control of the hot conduction wall. It can be seen that the average temperature of water instantly responds to the high temperature of heat source and leads to a sharp increase of its temperature to nearly $450 \mathrm{~K}$ in a short time. As time goes on, the average temperature of water begins to slow down at a relatively slower speed. Note that there is a plunge in the range of $0.3-0.4 \mathrm{~ns}$ in the curves. It can be analyzed that this phenomenon may be arriving at the cold side of the cluster of water

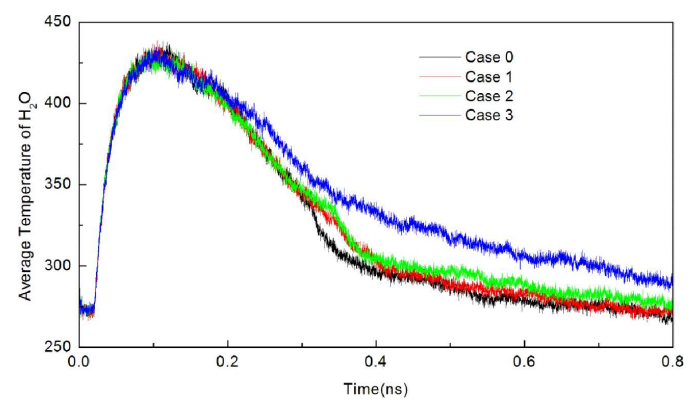

FIG. 2: Average temperatures of water molecules in cases with and without nitrogen 
molecules, after which the temperature of the cluster rapidly decreases with the condensation at the cold surface. After the plunge, the temperatures decrease with further slower speed.

Comparing with the pure water in Case 0, the introduction of nitrogen in the working fluid region helps to raise the average temperature of working media. The average temperature of water molecules increases gradually with the increase in the number of nitrogen molecules (from Case 0 to Case 3). To some extent, higher temperature difference means more thermal resistance between substances. The existence of many nitrogen molecules resists the heat transfer between water molecules and the cold wall to increase the temperature difference. The curve of temperature variation in Case 1 shows a similar tendency with the curve in Case 2, which is a little higher than the data in Case 0 . With the further increase of the number of nitrogen, the average temperature of water molecules increases significantly. Furthermore, the plunge in the range of 0.3-0.4 ns in Case 3 shows an obvious gentle tendency, which demonstrates the noticeable impact of the nitrogen molecules to the thermal resistance between water molecules and cold wall.

The increase and decrease of the average temperature of water molecules can demonstrate that vaporization and condensation play a primary role, respectively, during the whole phasechange process. The liquid water molecules initially placed at the bottom of the working fluid region are heated first. At the same time, the vapor water molecules may move in the vapor region and collide with the liquid water molecules at the interface to obtain the heat or run into the liquid water region. With further heating by the conduction wall adjacent with the heat source, liquid water molecules at the upper positions overcome the intermolecular attraction and start to vaporize. The water molecules in the vapor state separate from the liquid water may rise and continue moving to other locations.

In the case of pure water, the collision between water molecules during the course of the movement results in the continuous transportation of thermal energy between the water molecules and the increase of water temperature. The thermal energy transport between various water molecules continues until the water molecules with high energy condense on the lower surface of the conduction wall at the cold side and release the remaining energy to the cold wall. More and more water molecules continuously accumulate near the conduction wall of the cold side, and the corresponding number of water molecules being heated at the hot side gradually decrease. Therefore, the average temperature of the water begins to slowly decline. Since the condensation of most vapor water molecules transformed from liquid water takes place after they pass through a certain distance of the working fluid region, the process of decreasing the average temperature is slower than the steep increase process. In the case of water with nitrogen, the collision between the water molecules and nitrogen molecules occurs at the same time. The nitrogen molecules get part of the energy from water molecules, which will not transfer to the cold wall through condensation. On the other hand, the accumulation of partial nitrogen molecules near the cold conduction wall reduces the condensation of water. Both of these effects lead to the relatively higher average temperature compared to the case without nitrogen.

Figures 3(a) and 3(b) show the variations of the number of vapor and liquid water molecules for the four cases with different numbers of nitrogen molecules, respectively. For the pure water case, the instantly heated liquid molecules start to leave the initially liquid region and move into the vapor region, leading to a rapid increase of the number of vapor water at the beginning. At the same time of the vaporization, the condensation at the cold wall side occurs when the higher temperature vapor molecules transfer energy to the lower temperature copper wall. From the beginning to $0.2 \mathrm{~ns}$, vaporization may play a primary role because the number of molecules of the vapor phase changing from the liquid phase is much larger than that of liquid molecules changing from the vapor phase, which leads to the sustained growth of vapor water number.

Volume 27, Issue 1, 2020 


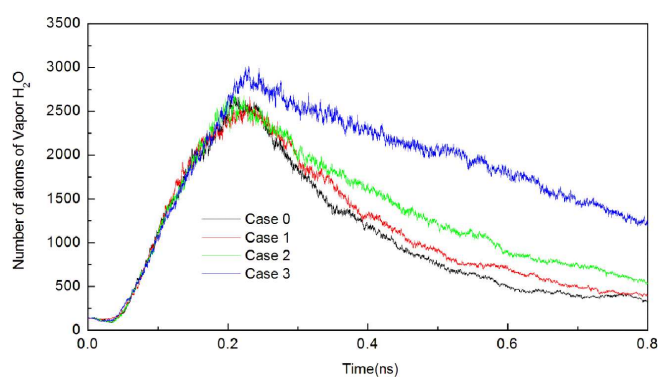

(a)

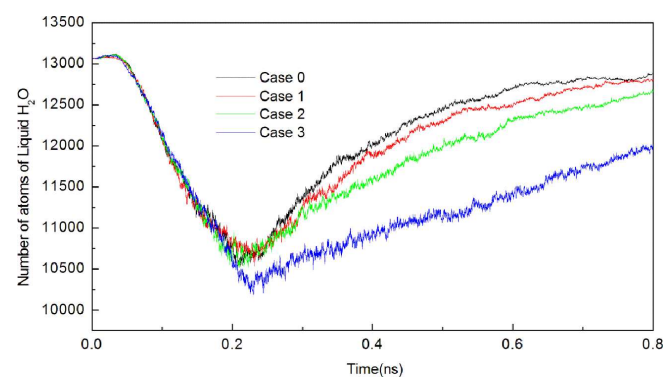

(b)

FIG. 3: Number of water molecules in cases with and without nitrogen: (a) vapor water, (b) liquid water

Then the number of vapor molecules starts to decrease due to the condensation of more and more vapor molecules at the cold side after $0.2 \mathrm{~ns}$. The tendency of decrease gradually slows down because of the coverage of condensed water on the lower surface of the cold conduction wall and the substantial reduction of liquid water molecules at the hot side. The amount of vapor water under the nitrogen condition basically results in a higher value than that of the case without nitrogen at the corresponding time. On the other hand, the curves of the number of liquid water present the opposite rule of increasing and decreasing because of the constant total number of water molecules, as shown in Fig. 3(b). Combined with the influence of nitrogen to the number of liquid or vapor water, the influence of heat transfer can be further observed.

Figure 4(a) shows the $y-z$ projections of the simulation domain in the case without nitrogen at different times. The initial subfigure shows the first construction of the simulation domain. The section of vacuum space was left out to keep the same height of work fluid region in the four cases. After steps of relaxation and equilibrium, the molecules changed their locations as shown in the subfigure of $0.02 \mathrm{~ns}$. With the heating of the heat source, the vaporization starts from the upper surface of the liquid region. Some of the liquid molecules leave the liquid region and move into the vapor region. On the other hand, a few vapor molecules begin to condense on the surface of the cold conduction wall at the top side. The number of liquid water molecules decreases gradually, while that of vapor water molecules increases. A cluster of water molecules was divorced from the hot conduction wall and driven to the cold conduction wall by the heating directly because of the heat wall's high temperature, which can be seen from $0.12 \mathrm{~ns}$. After $0.17 \mathrm{~ns}$, it can be seen clearly that the number of water molecules at the hot end decreases, while the value at the cold end increases. Combining this group of snapshots demonstrates the turning point of the curves in Fig. 2, which corresponds to the cluster apart from the heat wall side near the time of $0.1 \mathrm{~ns}$. Besides, the plunge of the temperature between $0.3 \mathrm{~ns}$ and $0.4 \mathrm{~ns}$ corresponds to the processing of the molecules cluster arriving at the cold wall side. Moreover, with the gradual decrease of the number of vapor molecules between the hot and cold ends, the total number of liquid molecules that increases slowly after a period of time can be easily understood.

Figure 4(b) shows the $y$ - $z$ projections near the lower surface of the cold conduction wall in the pure water case at the corresponding time in Fig. 4, which helps to understand the distribution of water molecules near the cold conduction wall. There are no water molecules on the cold conduction wall at first. As time goes on, the liquid water molecules condense at the cold conduction wall and the number of the condensed molecules increases. After $0.22 \mathrm{~ns}$, the lower surface of conduction wall is nearly covered by the condensed water. 


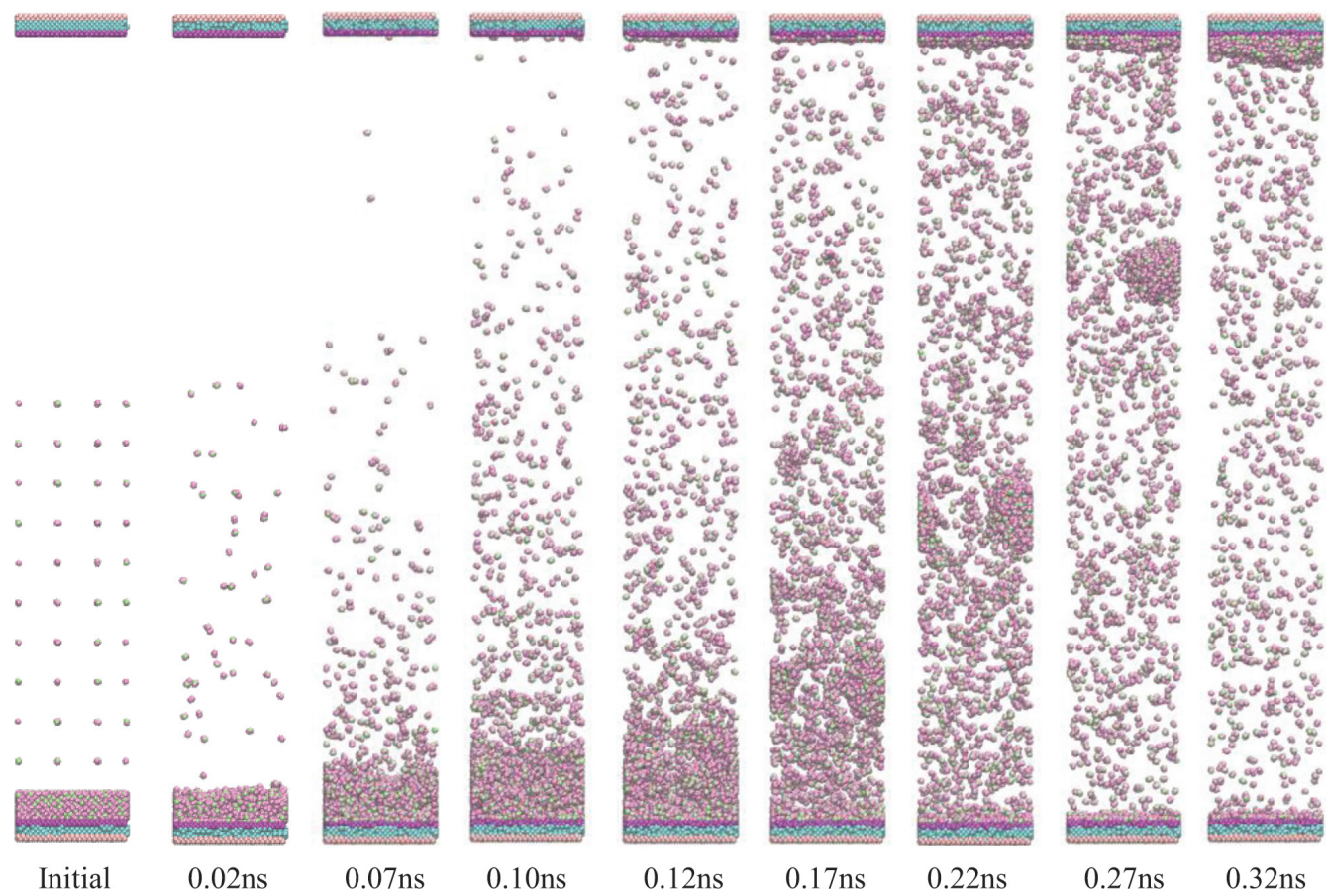

(a)

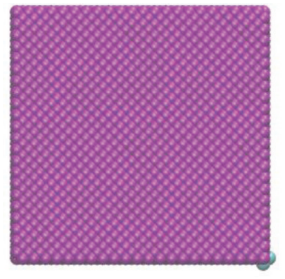

Initial

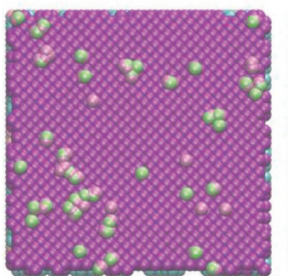

$0.12 \mathrm{~ns}$

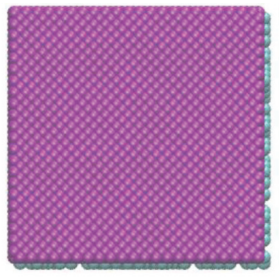

$0.02 \mathrm{~ns}$

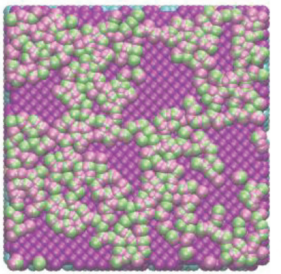

$0.17 \mathrm{~ns}$

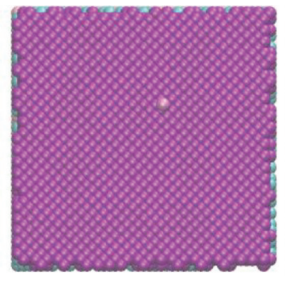

$0.07 \mathrm{~ns}$

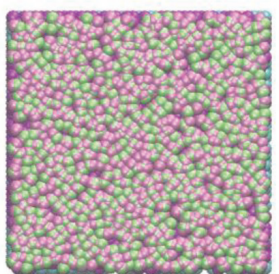

$0.22 \mathrm{~ns}$

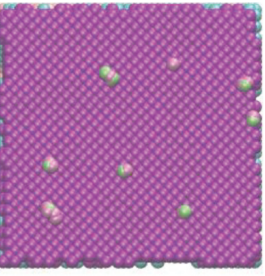

$0.10 \mathrm{~ns}$

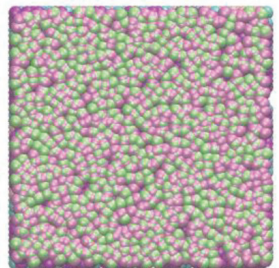

$0.32 \mathrm{~ns}$

(b)

FIG. 4: Snapshots of Case 0: (a) in $y-z$ projection, (b) in $x-y$ projection on the cold conduction wall

Figures 5(a) and 5(b) show the $y$ - $z$ projections and $x-y$ projections of simulation domains of Case 1 from initial to $0.32 \mathrm{~ns}$, respectively. The nitrogen molecules initially distributed on the upper section of the working fluid region. Comparing Figs. 4(a) and 5(a) after 0.12 ns, it can be 


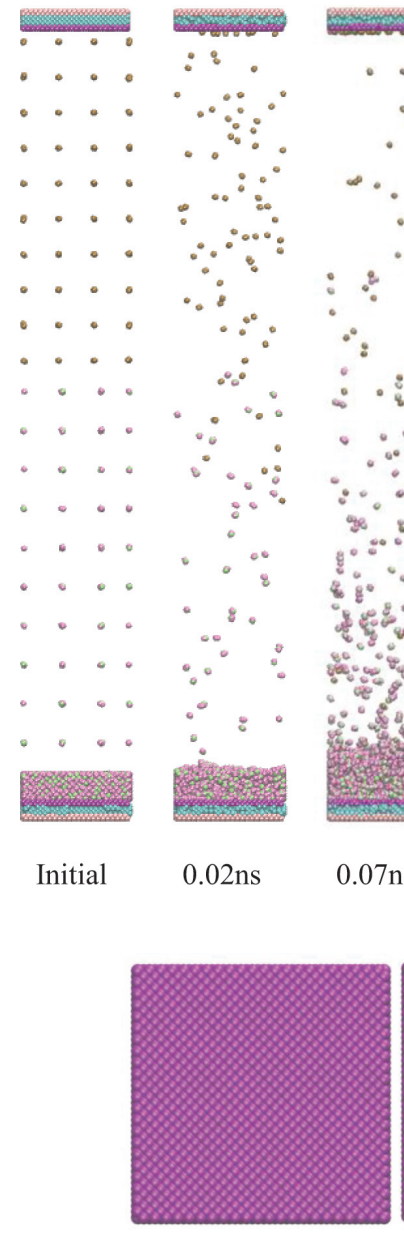

Initial

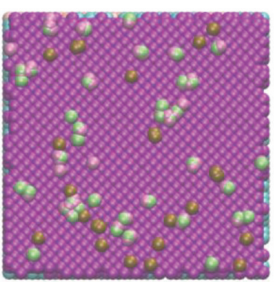

$0.12 \mathrm{~ns}$

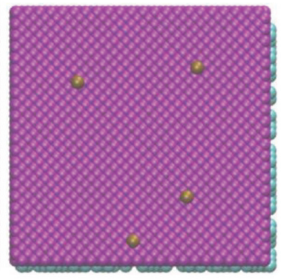

$0.02 \mathrm{~ns}$

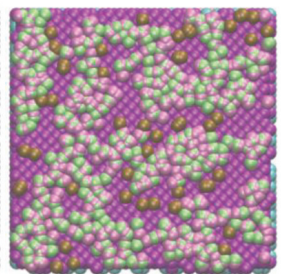

$0.17 \mathrm{~ns}$

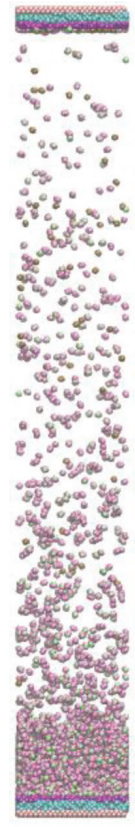

$0.12 \mathrm{~ns}$

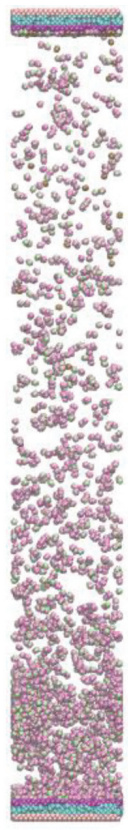

$0.17 \mathrm{~ns}$

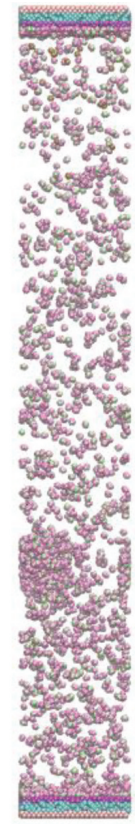

$0.22 \mathrm{~ns}$
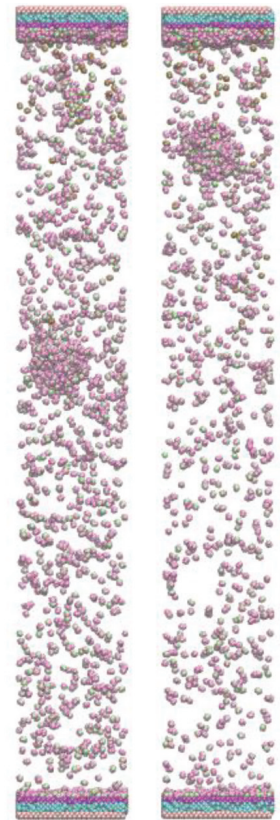

$0.27 \mathrm{~ns}$

$0.32 \mathrm{~ns}$

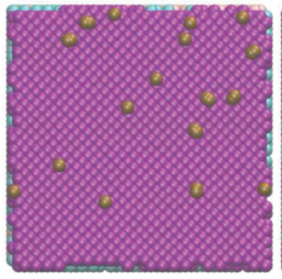

$0.07 \mathrm{~ns}$

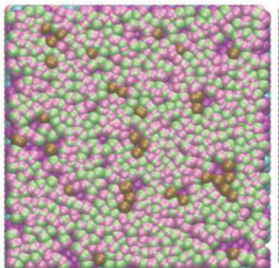

$0.22 \mathrm{~ns}$

(b)

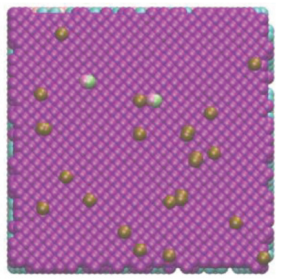

$0.10 \mathrm{~ns}$

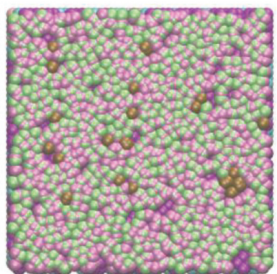

$0.32 \mathrm{~ns}$

FIG. 5: Snapshots of Case 1: (a) in $y$ - $z$ projection, (b) in $x-y$ projection on the cold conduction wall

seen that the velocity of the water molecules cluster moving and aggregating on the cold wall is slower than that of the pure water case. Comparing Fig. 5(b) to Fig. 4(b) before 0.12 ns, a number of nitrogen molecules gathering on the cold wall happened earlier than some water molecules. 
After $0.12 \mathrm{~ns}$, it can be seen that the number of water molecules aggregating on the cold wall is smaller than those without nitrogen. As shown in the subfigures in Fig. 5(b), there are still some regions of cold that cannot be covered by the liquid water molecules until $0.32 \mathrm{~ns}$ because the existence of nitrogen occupied some space of the cold wall to resist the condensation of water. The nitrogen molecules play a major role in resisting the condensation phase change of vapor water.

\subsection{Distribution and Trajectories of Molecules}

In order to further understand the variation of the molecules quantitatively, it is important to investigate the spatial distribution of water molecules and nitrogen molecules. Figure 6(a) shows the mass density of water molecules distributed along the $z$ direction in the case of pure water and with nitrogen as time goes on. The evolution of phase change near the hot wall and cold wall in the four cases could be clearly seen in Fig. 6(a) according to the variation of density. The density curves generally show the movement of the peak, which means the location of cluster in the processing. The space outside of the peaks section in the region is full of vapor molecules with low values of density. At the time of $0.02 \mathrm{~ns}$, a large number of water molecules crowd near the hot end (dimensionless height equals 0.0 ), which demonstrates the aggregation of liquid water. With the beginning of condensation on the cold surface, the mass density of water molecules near the cold wall (dimensionless height equals 1.0) gradually appears and increases. Meanwhile, the mass density of water molecule layers near the hot wall decreases. From the location of peak at different times, it can be seen that the liquid cluster moves from the hot end to the cold end. In time, the peak formed by the water cluster moves to the high position until it arrives at the cold wall. For the cases with nitrogen, the peak moves slightly slower than Case 0 , without nitrogen, because of the occupation of the nitrogen molecules. At $0.32 \mathrm{~ns}$, the large number of water molecules crowd near the cold side in the case of no nitrogen, but the cluster in the cases with nitrogen is far away from the cold wall. The height of the peaks has a slight decline with the progress of simulation except for the locations near the two walls, which indicates some water molecules around the cluster may move from the cluster to the vapor region gradually through collision and diffusion in the course of movement.

Aiming to investigate the attribute of nitrogen molecules, Fig. 6(b) shows the mass density of nitrogen in the working fluid region. It can be seen that the density of nitrogen is far less than the water molecules. From the density curve of nitrogen, it can be found that the variation of nitrogen has an interesting rule. A great number of nitrogen molecules accumulates near the cold wall as time goes on, while a certain amount of nitrogen molecules continuously keep moving as noncondensable gas diffusion in the whole working fluid region. The movement of nitrogen gets wide distribution in the whole working fluid region but does not concentrate in a small range unlike water molecules. The existence of nitrogen has two functions-the accumulated molecules near the cold wall could enhance the thermal resistance, and the movement of molecules may increase the opportunity of collision between various molecules, leading to the reduction of heat transfer between the water and cold wall.

To research the path of the working fluid molecules in the process of phase change, several molecules were randomly chosen to track their trajectories and the detailed data of their coordinates were obtained. Five water molecules (H2O_L1, H2O_L2, H2O_V1, H2O_V2, and H2O_V3) and five nitrogen molecules (N2_1, N2_2, N2_3, N2_4, and N2_5) at different initial positions were labeled in the initial subfigure in Fig. 7. Remember that the labeled molecules were named according to their initial locations, in which two molecules (H2O_L1 and H2O_L2)

Volume 27, Issue 1, 2020 

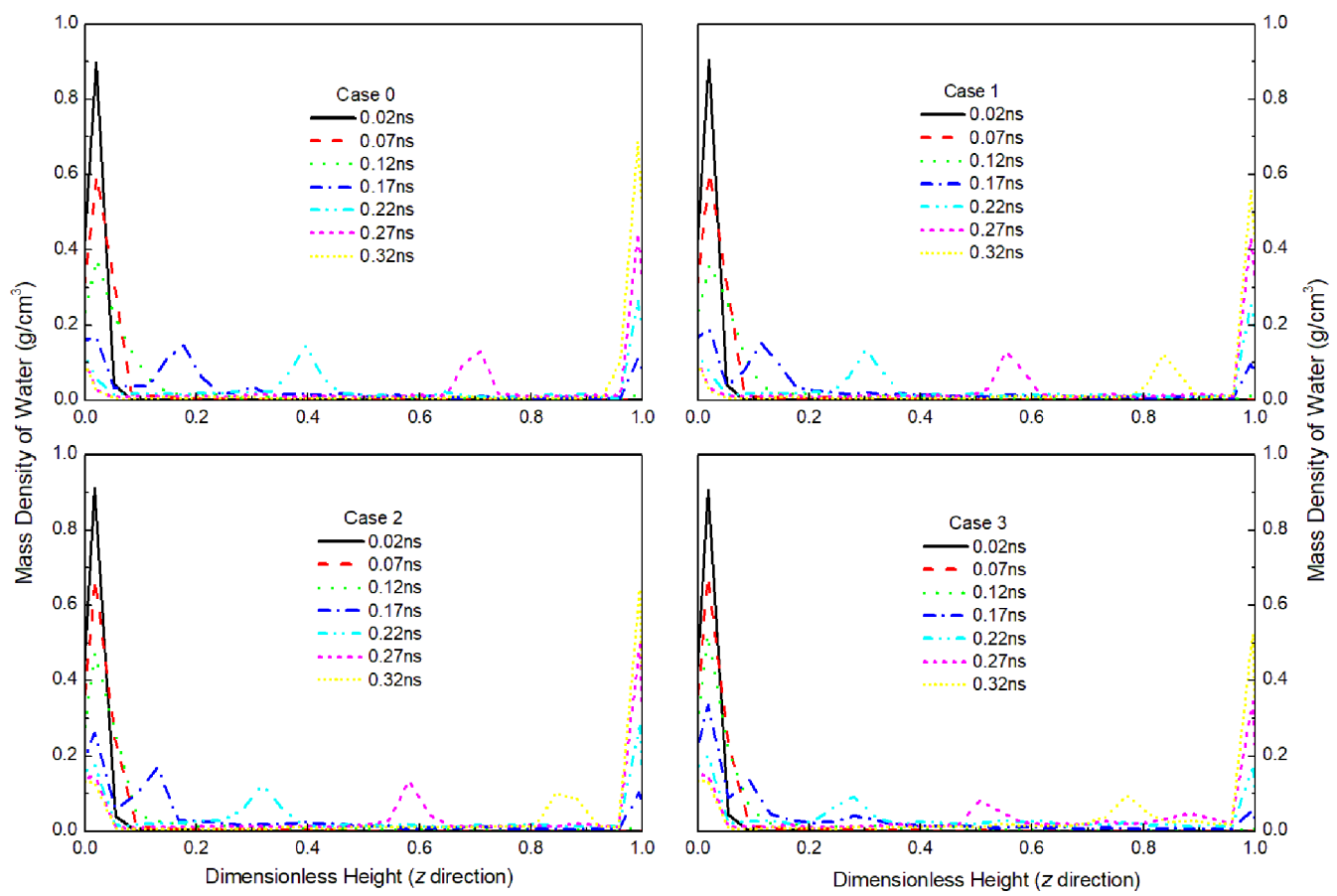

(a)

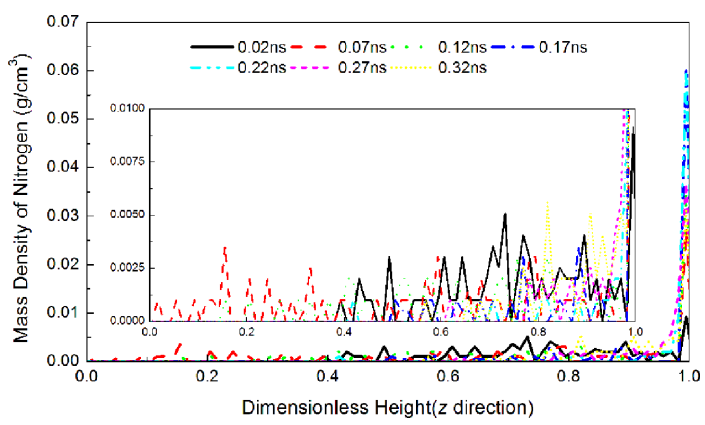

(b)

FIG. 6: Mass density of molecules: (a) mass density of water molecules in cases with and without nitrogen, (b) mass density of nitrogen molecules in Case 1

locate in the liquid water region and three molecules (H2O_V1, H2O_V2 and H2O_V3) locate in the vapor water region at the initial time. In the course of the transportation, the water molecule phase would change but the labeled name remained unchanged in the subfigures to avoid confusion. The latter subfigures in Fig. 7 show the $y-z$ projections of the simulation domain with the 10 molecules labeled respectively as time passes. It can be seen from the subfigures that at different times, the movement of the molecules occurs in the whole working fluid region. All the labeled water molecules condense at the top side after a period of time whereas some nitrogen molecules still keep in motion in the whole region between the bottom side and the top side without specific laws. 


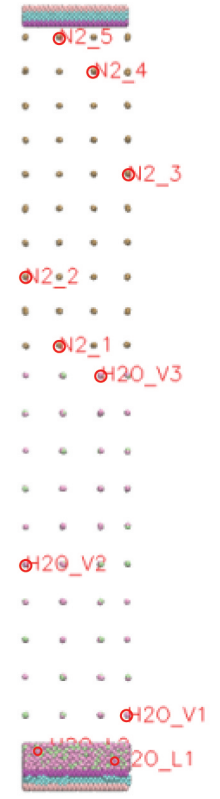

Initial

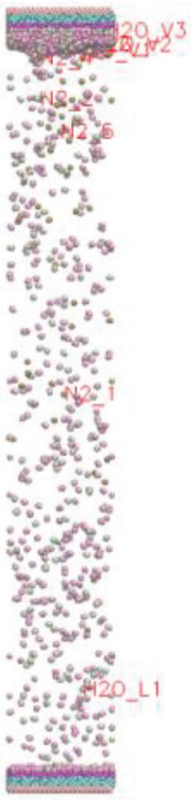

$0.42 \mathrm{~ns}$

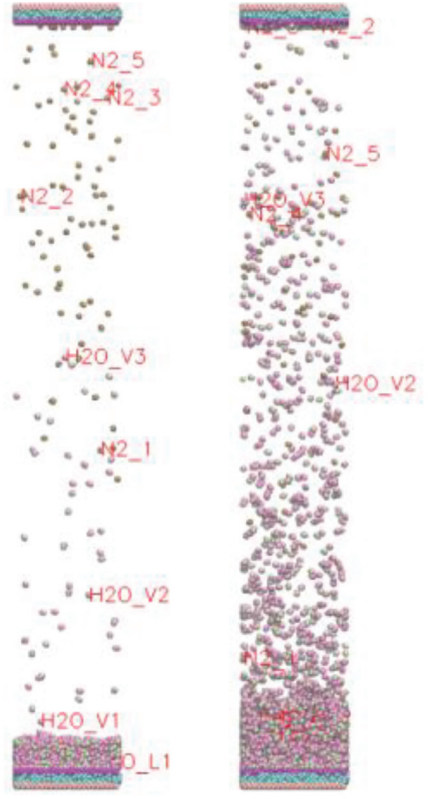

$0.02 \mathrm{~ns}$

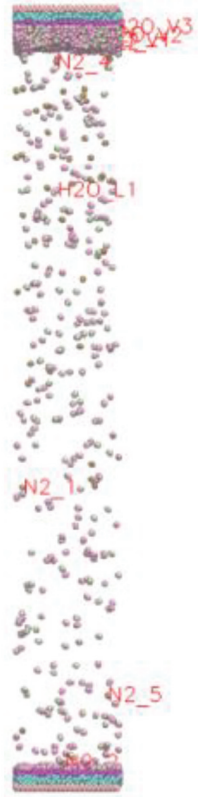

$0.52 \mathrm{~ns}$

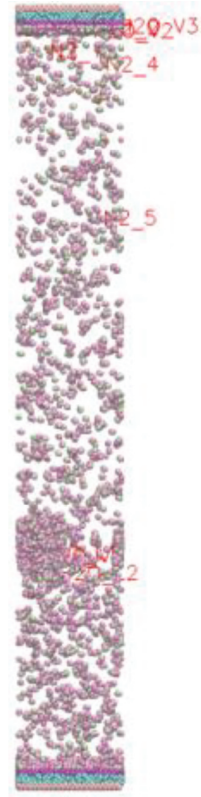

$0.22 \mathrm{~ns}$
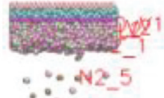

$\because 0^{\circ}$

$4 x_{2} 40$

$\because$ in

$\because \because \because$

$\therefore$

$\therefore \div$

$\therefore \div$

$\because \because$

$\because \cdots$

$\because \therefore \because$

$\because \because \because$

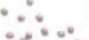

$0.0 \%$

$\because \because \frac{0}{0}$

$\therefore \because$

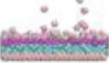

$0.72 \mathrm{~ns}$

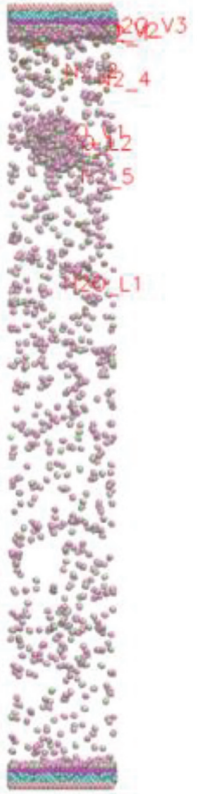

$0.32 \mathrm{~ns}$

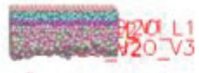

$\therefore$

- 2

- 8

$\therefore \cos ^{2}$

N2 $5 \cdot .0^{N} 2$

$\because x_{0} \dot{2}_{-1}$

$\therefore$

$\because$

$\because$

$\therefore \therefore 0^{N 2}-4$

$\therefore \therefore$

$\therefore \therefore$

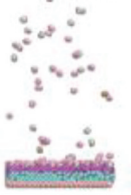

$0.82 \mathrm{~ns}$

FIG. 7: Snapshots of tracking trajectory of some molecules in Case 1

Figures 8(a) and 8(b) show the dimensionless height of these water and nitrogen molecules in the $z$ direction versus the time. From these curves it can be seen that the dimensionless heights of water molecules fluctuate between the vapor and liquid region for a short time until they arrive

Volume 27, Issue 1, 2020 


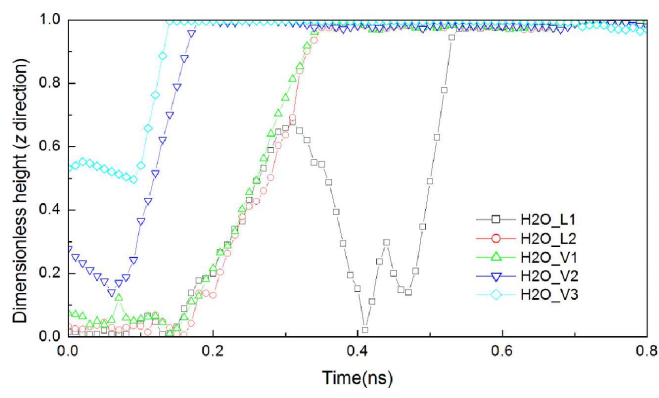

(a)

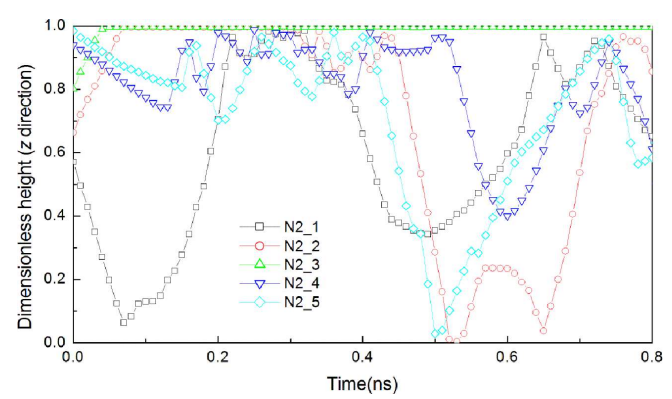

(b)

FIG. 8: Dimensionless height of $z$ direction of tracking trajectory of some molecules in Case 1: (a) water, (b) nitrogen

at the maximum location and vibrate near the cold conduction wall. Besides, it can be directly seen that some initial vapor water molecules (such as H2O_V1) occasionally entered the liquid region and then returned to the vapor region (between $0.1 \mathrm{~ns}$ and $0.2 \mathrm{~ns}$ ); some initial liquid water molecules returned to the liquid region again after moving up to the vapor region during a certain period of time (such as H2O_L1 around $0.41 \mathrm{~ns}$ ). The same vaporization and condensation phenomenon through the interface was introduced and discussed in the literature (Yang and Pan, 2005). On the other hand, the dimensionless heights of nitrogen fluctuate in the region from dimensionless height 0.0 to 1.0. Some nitrogen molecules (such as N2_3) vibrate near the cold wall and some of the labeled nitrogen molecules (such as N2_1, N2_2, N2_4, and N2_5) moved in and out of the liquid water region near the cold conduction wall and the vapor region with different amplitude of fluctuation. Generally speaking, from these trajectories of randomly selected molecules we get a glimpse of the characteristic that some water molecules may fluctuate in the liquid region and vapor region, which means the vaporization and condensation of water may change through the interface of vapor and liquid before they condense at the cold wall. At last, more and more water molecules condense at the cold wall, whereas some of the nitrogen molecules continuously fluctuate up and down in the whole working fluid region.

\section{CONCLUSIONS}

Molecular dynamics simulation was used to study the condensation in a confined region at nanoscale. Copper was chosen as the material of the hot wall and the cold wall. Water at liquid and vapor states were put between the hot and cold walls as the media of heat transfer. Cases without nitrogen and with nitrogen added in the working fluid region were designed to study the effect of noncondensable gases on condensation in confined space. The simulation results indicate that the presence of nitrogen helps to weaken the heat transfer of condensation because of the distribution and movement of nitrogen molecules. Some water molecules fluctuate in and out of the liquid and vapor regions, which means the vaporization and condensation of water may convert through the interface of vapor and liquid phases before they condense at the cold wall. Once the water molecules arrive at the cold wall they will condense and vibrate near the cold wall but no longer will move into the vapor region, whereas many nitrogen molecules continuously fluctuate up and down in the working fluid region to resist the heat transfer all the time without condensation. 


\section{ACKNOWLEDGMENTS}

The financial support for this research project from the National Basic Research Program of China (973 Program; Grant No. 2015CB251503) and the Fundamental Research Funds for the Central Universities (No. 2017MS019) are gratefully acknowledged.

\section{REFERENCES}

Abascal, J.L.F. and Vega, C., A General Purpose Model for the Condensed Phases of: TIP4P/2005, J. Chem. Phys., vol. 123, p. 234505, 2005.

Arismendi-Arrieta, D., Medina, J.S., Fanourgakis, G.S., Prosmiti, R., and Delgado-Barrio, G., Simulating Liquid Water for Determining Its Structural and Transport Properties, Appl. Radiat. Isotopes, vol. 83, pp. 115-121, 2014.

Cahill, D.G., Ford, W.K., Goodson, K.E., Mahan, G.D., Majumdar, A., Maris, H.J., Merlin, R., and Phillpot, S.R., Nanoscale Thermal Transport, J. Appl. Phys., vol. 93, pp. 793-818, 2003.

Diaz, R. and Guo, Z., Molecular Dynamics Study of Wettability and Pitch Effects on Maximum Critical Heat Flux in Evaporation and Pool Boiling Heat Transfer, Numer. Heat Transf., Part A, vol. 72, pp. 891-903, 2017a.

Diaz, R. and Guo, Z., A Molecular Dynamics Study of Phobic/Philic Nano-Pattering on Boiling Heat Transfer, Heat Mass Transf., vol. 53, pp. 1061-1071, $2017 \mathrm{~b}$.

Diaz, R. and Guo, Z., Enhanced Conduction and Pool Boiling Heat Transfer on Single-Layer GrapheneCoated Substrates, J. Enhanced Heat Transf., vol. 26, pp. 127-143, 2019.

Edom, A. and Vlassov, V., Experimental Study on Periodical Fluctuations of the Diffuse Vapor-Gas Front during Operation of a Gas-Loaded Heat Pipe, SAE Tech. Paper 2001-01-2234, 2001.

Edwards, D.K. and Marcus, B.D., Heat and Mass Transfer in the Vicinity of the Vapor-Gas Front in a Gas-Loaded Heat Pipe, ASME J. Heat Transf., vol. 94, pp. 155-162, 1972.

$\mathrm{Hu}, \mathrm{H}$. and Sun, Y., Effect of Nanopatterns on Kapitza Resistance at a Water-Gold Interface during Boiling: A Molecular Dynamics Study, J. Appl. Phys., vol. 112, p. 053508, 2012.

Huang, J., Zhang, J., and Wang, L., Review of Vapor Condensation Heat and Mass Transfer in the Presence of Non-Condensable Gas, Appl. Therm. Eng., vol. 89, pp. 469-484, 2015.

Jia, T., Zhang, Y., Ma, H.B., and Chen, J.K., Investigation of the Characteristics of Heat Current in a Nanofluid based on Molecular Dynamics Simulation, Appl. Phys. A-Mater., vol. 108, pp. 537-544, 2012.

Kim, N., Steam Condensation Enhancement and Fouling in Titanium Corrugated Tubes, J. Enhanced Heat Transf., vol. 26, pp. 59-74, 2019.

Lee, K., Kadambi, J.R., and Kamotani, Y., The Influence of Non-Condensable Gas on an Integral Planar Heat Pipe Radiators for Space Applications, Int. J. Heat Mass Transf., vol. 110, pp. 496-505, 2017.

Leriche, M., Harmand, S., Lippert, M., and Desmet, B., An Experimental and Analytical Study of a Variable Conductance Heat Pipe: Application to Vehicle Thermal Management, Appl. Therm. Eng., vol. 38, pp. 48-57, 2012.

Li, L., Ji, P., and Zhang, Y., Molecular Dynamics Simulation of Condensation on Nanostructured Surface in a Confined Space, Appl. Phys. A-Mater., vol. 122, p. 496, 2016.

Li, M., Huber, C., Mu, Y., and Tao, W., Lattice Boltzmann Simulation of Condensation in the Presence of Noncondensable Gas, Int. J. Heat Mass Transf., vol. 109, pp. 1004-1013, 2017.

Liang, Z., Biben, T., and Keblinski, P., Molecular Simulation of Steady-State Evaporation and Condensation: Validity of the Schrage Relationships, Int. J. Heat Mass Transf., vol. 114, pp. 105-114, 2017.

Volume 27, Issue 1, 2020 
Louden, P., Schoenborn, R., and Lawrence, C.P., Molecular Dynamics Simulations of the Condensation Coefficient of Water, Fluid Phase Equilib., vol. 349, pp. 83-86, 2013.

Mao, Y. and Zhang, Y., Molecular Dynamics Simulation on Rapid Boiling of Water on a Hot Copper Plate, Appl. Therm. Eng., vol. 62, pp. 607-612, 2014.

Morshed, A.K.M.M., Paul, T.C., and Khan, J.A., Effect of Nanostructures on Evaporation and Explosive Boiling of Thin Liquid Films: A Molecular Dynamics Study, Appl. Phys. A, vol. 105, pp. 445-451, 2011.

Nagayama, G., Kawagoe, M., Tokunaga, A., and Tsuruta, T., On the Evaporation Rate of Ultra-Thin Liquid Film at the Nanostructured Surface: A Molecular Dynamics Study, Int. J. Therm. Sci., vol. 49, pp. 59-66, 2010.

Péreza, A. and Rubiob, A., A Molecular Dynamics Study of Water Nucleation Using the TIP4P/2005 Model, J. Chem. Phys., vol. 135, p. 244505, 2011.

Ren, B., Zhang, L., Cao, J., Xu, H., and Tao, Z., Experimental and Theoretical Investigation on Condensation inside a Horizontal Tube with Noncondensable Gas, Int. J. Heat Mass Transf., vol. 82, pp. 588-603, 2015.

Rohani, A.R. and Tien, C.L., Steady Two-Dimensional Heat and Mass Transfer in the Vapor-Gas Region of a Gas-Loaded Heat Pipe, ASME J. Heat Transf., vol. 95, pp. 377-382, 1973.

Seyf, H.R. and Zhang, Y., Molecular Dynamics Simulation of Normal and Explosive Boiling on Nanostructured Surface, J. Heat Transf., vol. 135, p. 121503, 2013.

Sugimoto, K., Asano, H., Murakawa, H., Takenaka, N., Nagayasu, T., and Ipposhi, S., Evaluation of Liquid Behavior in a Variable Conductance Heat Pipe by Neutron Radiography, Nucl. Instrum. Methods Phys. Res. A, vol. 651, pp. 264-267, 2011.

Wang, C.S., Chen, J.S., Shiomi, J., and Maruyama, S., A Study on the Thermal Resistance over SolidLiquid-Vapor Interfaces in a Finite-Space by a Molecular Dynamics Method, Int. J. Therm. Sci., vol. 46, pp. 1203-1210, 2007.

Yang, T.H. and Pan, C., Molecular Dynamics Simulation of a Thin Water Layer Evaporation and Evaporation Coefficient, Int. J. Heat Mass Transf., vol. 48, pp. 3516-3526, 2005.

Yi, Q., Tian, M., Yan, W., Qu, X., and Chen, X., Visualization Study of the Influence of Non-Condensable Gas on Steam Condensation Heat Transfer, Appl. Therm. Eng., vol. 106, pp. 13-21, 2016.

Yu, J. and Wang, H., A Molecular Dynamics Investigation on Evaporation of Thin Liquid Films, Int. J. Heat Mass Transf., vol. 55, pp. 1218-1225, 2012.

Zhang, C., Cheng, P., and Minkowycz, W.J., Lattice Boltzmann Simulation of Forced Condensation Flow on a Horizontal Cold Surface in the Presence of a Non-Condensable Gas, Int. J. Heat Mass Transf., vol. 115, pp. 500-512, 2017.

Zhou, L., Wang, L., Chong, D., Yan, J., and Liu, J., CFD Analysis to Study the Effect of Non-Condensable Gas on Stable Condensation Jet, Prog. Nucl. Energ., vol. 98, pp. 143-152, 2017.

Zou, Y., Huai, X., and Lin, L., Molecular Dynamics Simulation for Homogeneous Nucleation of Water and Liquid Nitrogen in Explosive Boiling, Appl. Therm. Eng., vol. 30, pp. 859-863, 2010. 